\title{
Efficient numerical techniques for modeling multicomponent ground-water transport based upon simultaneous solution of strongly coupled subsets of chemical components
}

\author{
Bruce A. Robinson ${ }^{\mathrm{a}, *}$, Hari S. Viswanathan ${ }^{\mathrm{b}}$, Albert J. Valocchi ${ }^{\mathrm{b}}$ \\ ${ }^{a}$ Earth and Environmental Sciences Division, Los Alamos National Laboratory, Los Alamos, NM 87545, USA \\ ${ }^{\mathrm{b}}$ Department of Civil and Environmental Engineering, University of Illinois at Urbana-Champaign, Urbana, IL 61801, USA
}

Received 30 December 1998; received in revised form 30 July 1999; accepted 11 August 1999

\begin{abstract}
An iterative solution technique for reactive transport problems is developed, which we call the selective coupling method, that represents a versatile alternative to traditional uncoupled iterative techniques and the fully coupled global implicit method. The chemical formulation studied allows a combination of equilibrium and kinetic reactions, and therefore is a more versatile model formulation than a purely equilibrium-based system. However, this is a very challenging system for obtaining an efficient numerical solution. Techniques that sequentially compute the concentrations of aqueous components possibly ignore important derivatives in the Jacobian matrix of the full system of equations. The selective coupling method developed here allows only the strongly coupled components to be solved together, and the transport iteration consists of solving groups of components simultaneously. We also develop a method denoted as coupled normalization to reduce the computational work and memory requirements for particular types of reactive transport problems. These approaches can result in computational savings relative to the global implicit method by achieving a similar iteration count while reducing the cpu time per iteration. More importantly, the memory requirements of the selective coupling technique are controlled by the maximum number of coupled components, rather than by the total number of components. For complex aqueous chemical systems and grids with a large number of nodes, memory efficiency is the characteristic that makes the selective coupling method particularly attractive relative to the global implicit method. A series of example cases illustrate the efficiency of the new approach. These test problems are also used to address the implementation issues surrounding the most efficient strategy for coupling the aqueous components when carrying out the chemical transport iteration. In-depth knowledge of the behavior of the chemical system is required to select an appropriate solution strategy. Published by Elsevier Science Ltd.
\end{abstract}

Notation
Variable
$\alpha_{i j}$
$A$
$\bar{A}$
$A_{\mathrm{s}}$
$b$
$\bar{B}$

\section{Notation}

Variable

Description

stoichiometric coefficient representing the number of moles of component $j$ in complex $i$ aqueous concentration of the electron acceptor ( $\mathrm{mol} / \mathrm{kg}$ water) solute mass storage per unit total volume for an aqueous component $\left(\mathrm{mol} / \mathrm{m}^{3}\right)$ surface area of mineral $\left(\mathrm{m}^{2} / \mathrm{m}^{3}\right.$ rock) decay coefficient $\left(\mathrm{h}^{-1}\right)$ solute mass storage per unit total volume for a vapor component $\left(\mathrm{mol} / \mathrm{m}^{3}\right)$

\footnotetext{
${ }^{*}$ Corrresponding author. Fax: 001-505-665-8737.

E-mail address: robinson@lanl.gov (B.A. Robinson)
}

uncomplexed concentration (mol/kg water) total aqueous concentration ( $\mathrm{mol} / \mathrm{kg}$ water) chemical formula for an aqueous component hydrodynamic dispersion tensor $\left(\mathrm{m}^{2} / \mathrm{s}\right)$ Damköhler number array of residuals advective mass flux of solute $\left(\mathrm{mol} / \mathrm{m}^{2}\right)$ vapor concentration ( $\mathrm{mol} / \mathrm{kg}$ air) activity coefficient rate constant for precipitation-dissolution reaction $\left(\mathrm{mol} / \mathrm{m}^{2} \mathrm{~s}\right)$

$k_{\mathrm{f}} \quad$ reaction rate in the forward direction for Example 1 (1/time) sorption mass transfer coefficient $(1 / \mathrm{h})$ reaction rate in the reverse direction for Example 1 (1/time) 
$K_{\mathrm{A}} \quad$ Monod half-maximum-rate concentration for electron acceptor (mol $/ \mathrm{kg}$ water)

$K_{\mathrm{D}} \quad$ sorption distribution coefficient $\left(\mathrm{cm}^{3} / \mathrm{g}\right)$

$K_{\mathrm{S}} \quad$ Monod half-maximum-rate concentration for electron donor ( $\mathrm{mol} / \mathrm{kg}$ water)

$K_{\text {sp }} \quad$ solubility product for precipitation/ dissolution reaction [the units are dependent on the number of aqueous species participating in the precipitation/dissolution reaction along with their stoichiometry)

1 subscript denoting liquid phase

$L \quad$ advection-dispersion operator $\left(\mathrm{mol} / \mathrm{m}^{3}\right)$

$m_{\text {b,init }} \quad$ initial biomass concentration ( $\mathrm{mol} / \mathrm{kg}$ rock)

$M \quad$ total immobile concentration ( $\mathrm{mol} / \mathrm{kg}$ rock)

$\hat{M} \quad$ chemical formula for an immobile component

$\mu_{\mathrm{p}} \quad$ stoichiometric coefficients associated with precipitation/dissolution reactions

$N_{\mathrm{c}} \quad$ number of aqueous components

$N_{\text {cell }} \quad$ number of elements/cells in the finite element grid

$N_{\text {con }} \quad$ number of connections in the finite element grid

$N_{\text {dof }} \quad$ number of degrees of freedom being solved for

$N_{\text {im }} \quad$ number of immobile components

$N_{\mathrm{v}} \quad$ number of vapor components

$N_{\mathrm{x}} \quad$ number of aqueous complexes

$\phi \quad$ porosity $\left(\mathrm{m}^{3}\right.$ voids $/ \mathrm{m}^{3}$ total)

$Q \quad$ volumetric flow rate in column (arbritrary units)

$Q_{\mathrm{p}} \quad$ reaction quotient [the units of the reaction quotient are dependent on the number of aqueous species participating in the precipitation/dissolution reaction along with their stoichiometry)

$\rho_{\mathrm{b}} \quad$ bulk rock density $\left(\mathrm{kg} / \mathrm{m}^{3}\right)$

$\rho_{1} \quad$ density of water $\left(\mathrm{kg} / \mathrm{m}^{3}\right)$

$R \quad$ kinetic reaction source-sink term $\left(\mathrm{mol} / \mathrm{m}^{3}\right)$

$S \quad$ aqueous concentration of substrate (electron donor) ( $\mathrm{mol} / \mathrm{kg}$ water)

$S_{1} \quad$ liquid saturation $\left(\mathrm{m}^{3}\right.$ water $/ \mathrm{m}^{3}$ voids)

$t \quad$ time (s)

$\tau \quad$ residence time of column (arbitrary units)

$u_{1} \quad$ Darcy velocity $(\mathrm{m} / \mathrm{s})$

$\mathrm{v} \quad$ subscript denoting vapor phase

$x \quad$ complex concentration ( $\mathrm{mol} / \mathrm{kg}$ water)

$\hat{X} \quad$ chemical formula for aqueous complex

$Y \quad$ microbial yield coefficient (g cell/mole NTA)

\section{Introduction}

Transport of dissolved chemicals in groundwater is an important topic in several disciplines of the field of hydrology, including pollutant migration and hydrogeochemical characterization of groundwater flow. Geochemical modeling takes many forms, including: (1) pure geochemical modeling without consideration of transport issues; (2) simulations of complex geochemical processes in one dimension; (3) contaminant plume migration in complex flow systems without consideration of the complexities of chemical reactions; and (4) models that combine complex reactive processes with detailed descriptions of groundwater flow. The recent trend in reactive transport modeling has been to include geochemical complexities such as biological reactions [13], explicit characterization of root zone processes [18], multiphase reactions such as mineral dissolution/precipitation reactions and their feedback on the flow system [10], and the influence of heterogeneities [21]. As the chemical aspects of these models become more complex, there has been considerable focus on numerical formulations that can solve the resulting equations efficiently. Steefel and MacQuarrie [17] provide a summary of these methods and approaches.

In most reactive transport codes, the transport of species is modeled using the advection-dispersion-reaction equation. The method by which transport codes incorporate the chemistry varies depending on the particular code. In general, the chemistry of the system can be modeled using a kinetic, equilibrium, or mixed kinetic-equilibrium formulation. The first codes that coupled complex chemistry with transport typically assumed that all chemical species in the system were in local equilibrium $[3,4,14,28]$. Papers by Yeh and Tripathi [28], Liu and Narasimhan [11] and Lichtner [10] review many of these models and discuss their approaches. Due to computational limitations and the virtually nonexistent database of mineral-water reaction rates, the local equilibrium assumption was a necessity. Equilibrium approaches remain popular due to the accessibility of large geochemical databases and sophisticated software packages. Nevertheless, the current trend in reactive transport codes is to include kinetic formulations for certain types of chemistry. Experimental evidence of kinetic limitations is shown in $[26,2,12,23,6,7]$. In addition, there now exists a considerable body of knowledge of mineral kinetic rate constants [16]. Therefore, kinetic formulations are useful for these water-rock interactions since both kinetic and equilibrium behavior can be modeled with a kinetic formulation [16].

Various approaches have been taken to model combined kinetic and equilibrium transport systems. We will refer to these approaches as mixed equilibrium-kinetic formulations. The equations that give rise to these kinetic formulations can be cast so that the minimum number of independent unknowns are solved for while still incorporating the appropriate kinetic reactions. For example, Liu and Narasimhan [11] include kinetic 
precipitation-dissolution in their otherwise equilibrium transport code. Steefel and Lasaga [16] and TebesStevens et al. [20] assume that aqueous phase reactions are in local equilibrium but include kinetic models for rock-water interactions. We will utilize a mixed equilibrium-kinetic formulation for the work presented in this paper.

The coupled partial differential equations that arise in a reactive transport formulation are computationally expensive to solve. There are numerous solution techniques that have been presented in the literature that deal with the problem of how to couple the reaction and transport terms. These techniques can be broken down into three categories: operator splitting, the global implicit method, and sequential iteration approaches. Each technique has its advantages and disadvantages in both accuracy and computational efficiency. Steefel and MacQuarrie [17] compare these techniques in greater detail. The reactive transport model presented in this paper utilizes a solution technique that is a hybridized version of the global implicit and sequential iteration methods presented in the literature. We will compare the performance of our method to the global implicit and sequential iteration methods. Comparisons between our method and operator-splitting methods are not discussed in this paper but will be a topic for future work.

The focus of the present study is to demonstrate a numerical technique that can be used to solve the mixed equilibrium-kinetic transport problem in large, complex two- and three-dimensional domains. The goal is to develop a flexible technique that has wide applicability to a range of reactive transport systems, with particular emphasis on the solution of transport problems with large number of numerical grid blocks. Therefore, both cpu time and memory usage must be handled efficiently. A technique is developed in an existing finite element heat and mass transfer code called FEHM [31], which handles multiphase flow and transport and uses a versatile finitevolume discretization approach capable of handling unstructured grids. Therefore, the techniques are applicable for use on grids of arbitrary complexity, from simple orthogonal grids to grids with node points having variable number of connections to adjacent nodes.

\section{Formulation of physical/chemical system}

\subsection{Primary/secondary variables}

The reactive transport equations solved by FEHM are described in greater detail in Viswanathan et al. [25]. We will provide a brief summary in this section. FEHM uses aqueous, immobile and vapor components as the primary dependent variables (PDVs) in the reactive transport equations. The secondary dependent variables (SDVs) are uncomplexed aqueous component concen- trations and aqueous complex concentrations. We make the local equilibrium assumption for all aqueous phase speciation reactions, resulting in the following relationship between PDVs and SDVs:

$C_{j}=c_{j}+\sum_{i=1}^{N_{\mathrm{x}}} \alpha_{i j} x_{i}, \quad j=1, \ldots, N_{\mathrm{c}}$,

where $C_{j}$ is the total aqueous concentration of component $j, c_{j}$ the uncomplexed concentration of component $j, x_{i}$ the concentration of complex $i, \alpha_{i j}$ the stoichiometric coefficient representing the number of moles of component $j$ in complex $i, N_{\mathrm{c}}$ the number of aqueous components and $N_{\mathrm{x}}$ is the number of aqueous complexes [28].

For transient fluid flow, the generalized version of the reactive transport equation for an aqueous component is used:

$$
\begin{aligned}
& \frac{\partial \bar{A}_{j}}{\partial t}=\nabla \cdot\left(\phi S_{1} D_{j} \nabla \rho_{1} C_{j}\right)-\nabla \cdot f_{\mathrm{c}}+R_{j} \\
& \quad j=1, \ldots, N_{\mathrm{c}}
\end{aligned}
$$

where $\bar{A}_{j}=\phi C_{j} S_{1} \rho_{1}$ is the solute mass storage per unit total volume for aqueous component liquid concentration $C_{j} ; f_{\mathrm{c}}=\rho_{1} C_{j} u_{1}$ the advective mass flux of solute; $D$ the hydrodynamic dispersion tensor; $S_{1}$ the liquid saturation; $u_{1}$ the Darcy velocity vector; $\phi$ the porosity; $\rho_{1}$ the liquid density; and $R_{j}$ is the kinetic reaction sourcesink term. To simplify the notation for the remainder of the paper, we define the advection-dispersion operator

$L_{1}(C)=\nabla \cdot f_{\mathrm{c}}-\nabla \cdot\left(\phi S_{1} D_{j} \nabla \rho_{1} C_{j}\right)$.

Eq. (2), the reactive advection-dispersion equation, can then be rewritten as

$\frac{\partial \bar{A}_{j}}{\partial t}+L_{1}\left(C_{j}\right)=R_{j}, \quad j=1, \ldots, N_{\mathrm{c}}$,

The reaction transport equation for a vapor component takes on a similar form to Eq. (4) and is given by

$\frac{\partial \bar{B}_{k}}{\partial t}+L_{\mathrm{v}}\left(G_{k}\right)=R_{k}, \quad k=1, \ldots, N_{\mathrm{v}}$,

where $\bar{B}_{k}$ is the solute mass of component $k$ per unit volume, $G_{k}$ the vapor concentration of component $k, u_{\mathrm{v}}$ the vapor Darcy velocity vector and $N_{\mathrm{v}}$ is the number of vapor components. The hydrodynamic dispersion tensor is assumed to reduce to longitudinal and transverse components (e.g. [10]).

Immobile components are not transported and are therefore treated using a simple mass balance given by

$\frac{\partial M_{m}}{\partial t}=R_{m}, \quad m=1, \ldots, N_{\text {im }}$,

where $M_{m}$ is the immobile concentration of component $m$ and $N_{\text {im }}$ is the number of immobile components. 
The reaction rate terms in Eqs. (4)-(6) originate from the kinetic reactions in the system and may be nonlinear functions of the total concentration of aqueous components, uncomplexed aqueous components, aqueous complexes, immobile components and vapor components. FEHM is capable of modeling the following kinetic processes: linear adsorption, nonlinear Langmuir adsorption, ion/surface exchange, precipitation-dissolution and liquid-vapor interchange of solute. The specific kinetic models that are available are described in the kinetic reaction types section. Eqs. (4)-(6) result in a system of $\left(N_{\mathrm{c}}+N_{\mathrm{im}}+N_{\mathrm{v}}\right)$ nonlinear coupled partial differential equations (PDEs). Discretizing these equations results in a set of $\left(N_{\mathrm{c}}+N_{\text {im }}+N_{\mathrm{v}}\right) N_{\mathrm{n}}$ algebraic equations where $N_{\mathrm{n}}$ is the number of spatial grid points. FEHM's method for solving this system of coupled PDEs will be discussed in the solution procedure section.

\subsection{Speciation reactions}

Given all of the aqueous component total concentrations, the uncomplexed aqueous component concentrations and aqueous complex concentrations can be calculated using chemical equilibrium theory. The chemical equilibrium calculations performed by FEHM are similar to the techniques used in batch geochemical software such as EQ3/6 [27]. A chemical reaction describing aqueous speciation can be written in the following general form

$\sum_{j=1}^{N_{\mathrm{c}}} \alpha_{i j} \hat{C}_{j} \Longleftrightarrow \hat{X}_{i}, \quad i=1, \ldots, N_{\mathrm{x}}$,

where $\hat{C}_{j}$ is the chemical formula for the aqueous component $j$, and $\hat{X}_{i}$ is the chemical formula for the aqueous complex $i$. FEHM assumes that all aqueous speciation reactions are at local equilibrium. The mass-action expression for an aqueous component is given by [19]

$K_{i}=x_{i} \prod_{j=1}^{N_{\mathrm{c}}}\left(\gamma_{j} c_{j}\right)^{-\alpha_{i j}}$

where $K_{i}$ is the equilibrium formation constant for complex $i$ and $\gamma_{j}$ is the activity coefficient for aqueous component $j$. In the present study, we neglect ionicstrength corrections. Eqs. (1) and (8) can be combined to express the total aqueous concentration of component $j$ as a function of the uncomplexed component concentrations:

$C_{j}=c_{j}+\sum_{i=1}^{N_{\mathrm{x}}} \alpha_{i j} K_{i} \prod_{z=1}^{N_{\mathrm{c}}} c_{z}^{\alpha_{i z}}, \quad j=1, \ldots, N_{\mathrm{c}}$,

Eq. (9) results in a set of $N_{\mathrm{c}}$ nonlinear algebraic equations to be solved given all of the total aqueous concentrations.

\subsection{Kinetic reaction types}

Kinetic reactions modeled by FEHM cannot be described by a single reaction rate expression. Rate expressions are available to simulate sorption, precipitation/dissolution, dual Monod biodegradation, and a general reversible reaction. Additional kinetic rate expressions are available, however, we will only discuss the kinetic rate expressions that are used in the test cases section of this paper. Note that the kinetic rate expressions do not necessarily conserve charge. In all of the reactive transport systems modeled in this paper, we do not attempt to model the complete set of chemical reactions, but attempt to model the chemical reactions that are relevant to the current study. Since all reactions are not included, a charge balance over the entire system cannot be calculated. However, the reactions that control the $\mathrm{pH}$ in Examples 2 and 3 are known, allowing for variable $\mathrm{pH}$ calculations to be performed.

The sorption models we use in the current study contain the same parameters that are measured in laboratory and field experiments for the various applications we have investigated. For this reason, we have chosen a linear kinetic sorption, and a kinetic ion-exchange model. The retardation of contaminants due to adsorption/desorption can be modeled with a linear kinetic sorption/desorption expression. The rate of adsorption/desorption of component $j$ is given by

$R_{j}=-k_{\mathrm{m}}\left(c_{j}-\frac{M_{j}}{K_{\mathrm{D}}}\right)$,

where $k_{\mathrm{m}}$ is the mass transfer coefficient, and $K_{\mathrm{D}}$ is the distribution coefficient. As $k_{\mathrm{m}} \rightarrow \infty$, this expression reduces to the linear equilibrium isotherm.

Biodegradation is an irreversible process in which bacteria oxidize an organic substrate to produce energy and biomass. In addition to biomass, the biodegradation process requires the presence of an electron acceptor (e.g. oxygen, nitrate, etc.) and nutrients (e.g. nitrogen and phosphorous). An example of a simplified biodegradation reaction is given by the following reaction [19]:

$$
\begin{aligned}
& \text { Substrate }+ \text { Electron Acceptor }+ \text { Nutrients } \\
& \rightarrow \text { cells }+\mathrm{CO}_{2}+\mathrm{H}_{2} \mathrm{O} .
\end{aligned}
$$

FEHM models the rate of biodegradation of a substrate with a multiplicative Monod model, given by [19]

$R_{\mathrm{S}}=-q_{m} m_{\mathrm{b}} \frac{[S]}{K_{\mathrm{S}}+[S]} \frac{[A]}{K_{\mathrm{A}}+[A]}$,

where $[S]$ is the aqueous concentration of substrate (i.e., the electron donor), $[A]$ the aqueous concentration of the electron acceptor, and $m_{\mathrm{b}}$ is the concentration of the immobile biomass. The parameter $q_{\mathrm{m}}$ is the maximum specific rate of substrate utilization, which represents the maximum amount of substrate that can be consumed 
per unit mass of bacteria per unit time. The parameters $K_{\mathrm{S}}$ and $K_{\mathrm{A}}$ are the Monod half-maximum-rate concentrations for the electron donor and electron acceptor, respectively. The rate of microbial growth is given by the synthesis rate (which is proportional to the rate of substrate degradation) minus a first-order decay rate

$R_{\text {cells }}=-Y R_{\mathrm{s}}-b\left(m_{\mathrm{b}}-m_{\mathrm{b}, \text { init }}\right)$,

where $Y$ is the microbial yield coefficient and $b$ is the first-order microbial decay coefficient. In the above equation, the assumption is made that the background conditions are sufficient to sustain a microbial population of a given size; therefore, the biomass concentration is not allowed to fall below its initial background concentration, $m_{\mathrm{b} \text {,init }}$.

A general reaction describing the precipitation/dissolution of a mineral $\mathrm{p}$ can be written in the following form:

$\hat{M}_{\mathrm{p}} \quad \Longleftrightarrow \quad \mu_{p 1} \hat{C}_{1}+\mu_{p 2} \hat{C}_{2}+\cdots+\mu_{p 2} \hat{C}_{N_{\mathrm{c}}}$,

where $\hat{M}$ is the chemical formula for the mineral, and $\mu_{p j}$ are stoichiometric coefficients. The equilibrium constant for this reaction is known as the solubility product. Since the activity of a pure solid is equal to one, the reaction quotient, $Q_{\mathrm{p}}$, is defined as

$Q_{\mathrm{p}}=\prod_{j=1}^{N_{\mathrm{c}}} c_{j}^{\mu_{p j}}$.

At equilibrium, $Q_{\mathrm{p}}$ is equal to the solubility product. The surface-controlled rate of precipitation/dissolution of a mineral is given by:

$\frac{\mathrm{d} R_{j}}{\mathrm{~d} t}=A_{\mathrm{s}} \mu_{j} k_{+}\left(1-\frac{Q_{\mathrm{p}}}{K_{\mathrm{sp}}}\right)$,

where $A_{\mathrm{s}}$ is the reactive surface area of the mineral, $k_{+}$ the rate constant, and $K_{\mathrm{sp}}$ is the solubility product [9]. With this equation, a mineral will precipitate when it is supersaturated and dissolve when it is undersaturated.

\section{Numerical solution techniques}

\subsection{Newton-Raphson formulation}

The reactive transport equations given by Eqs. (4)-(6) result in a set of nonlinear coupled PDEs. The numerical implementation of the transport step can be derived by rewriting Eqs. (4)-(6) in fully implicit time-discretized form:

$\frac{C_{j}^{n+1}-C_{j}^{n}}{\Delta t}+L\left(C_{j}^{n+1}\right)=R_{j}^{n+1}, \quad j=1, \ldots, N_{\mathrm{c}}$,

$\frac{G_{k}^{n+1}-G_{k}^{n}}{\Delta t}+L\left(G_{k}^{n+1}\right)=R_{k}^{n+1}, \quad k=1, \ldots, N_{\mathrm{v}}$,
$\frac{M_{m}^{n+1}-M_{m}^{n}}{\Delta t}=R_{m}^{n+1}, \quad m=1, \ldots, N_{\mathrm{im}}$,

where $n$ indicates the time step level. Reactive transport codes in the literature solve Eqs. (17)-(19) using either the global implicit, operator-splitting, or sequential iterative methods [17]. The global implicit method solves the transport and reaction step simultaneously. On the other hand, operator-splitting methods solve the transport and reaction steps in sequence without iteration [22]. Finally, the sequential iterative approaches iterate between the transport and reaction steps until a fully implicit solution is achieved. In this paper, we present a technique which is a hybridized version of the global implicit and sequential iteration methods. We have chosen an implicit method since many of the problems we are interested in take place over long time scales. Implicit methods are often more efficient for such problems because of their ability to take larger time steps [17]. Problems for modeling advection-dominated flows over shorter timescales would be better suited for operator-splitting methods [17].

The reaction rate terms in Eqs. (17)-(19) can be estimated using a Taylor series expansion to linearize the reaction rate term

$$
\begin{aligned}
R_{i}^{n+1, p+1}= & R_{i}^{n+1, p}+\sum_{j=1}^{N_{\mathrm{c}}}\left(\frac{\partial R_{i}}{\partial C_{j}}\right)^{n+1, p}\left(C_{j}^{n+1, p+1}-C_{j}^{n+1, p}\right) \\
& +\sum_{k=1}^{N_{\mathrm{v}}}\left(\frac{\partial R_{i}}{\partial G_{k}}\right)^{n+1, p}\left(G_{k}^{n+1, p+1}-G_{k}^{n+1, p}\right) \\
& +\sum_{m=1}^{N_{\mathrm{im}}}\left(\frac{\partial R_{i}}{\partial M_{m}}\right)^{n+1, p}\left(M_{m}^{n+1, p+1}-M_{m}^{n+1, p}\right)+\cdots,
\end{aligned}
$$

where $p$ is the iteration level and $i$ represents either an aqueous, vapor or immobile component.

The iterative methods in the literature differ in the degree to which terms in the summation of Eq. (20) are included in the linearized expressions approximating Eqs. (17)-(19). The fully coupled approach, called the global implicit method [16] uses all derivative terms in Eq. (20). This method, a full Newton-Raphson approximation, results in a large system of coupled linear equations stemming from the derivative terms of $R_{i}$ with respect to other aqueous, vapor, or immobile components. The approximate iterative approaches in the reactive transport literature often drop terms in the summations to decouple the linear equations into smaller equations sets that are solved sequentially. The sequential iterative approach described by Engesgaard and Kipp [4] and Kinzelbach et al. [8] ignore all derivative terms, using only $R_{i}^{n+1, p}$ in Eq. (20). We will refer to 
this method as the SIA-0 method. The SIA-1 method estimates the reaction term [19] as

$R_{i}^{n+1, p+1}=R_{i}^{n+1, p}+\left(\frac{\partial R_{i}}{\partial C_{i}}\right)^{n+1, p}\left(C_{i}^{n+1, p+1}-C_{i}^{n+1, p}\right)$.

The SIA-1 approach seeks to improve the estimate of $R_{i}^{n+1, p+1}$ by using the terms in the summations of Eq. (20) where $j=i, k=i$, and $m=i$. That is, for component $i$, the relationships with aqueous, vapor and immobile components other than $i$ are neglected, and the linear equations arising from the individual components are solved separately. We have found that the SIA-1 greatly improves convergence for large Damköhler number systems, when kinetics are fast compared to the transport time scale [19]. SIA-1 often outperforms SIA-0 because the $\Delta t \partial R_{i} / \partial C_{i}$ term is often significant, whereas, $\Delta t \partial R_{i} / \partial C_{j}, i \neq j$ terms are not. However, for certain reactions, the SIA-1 approach becomes quite inefficient because some of the cross-derivative terms $\left(\Delta t \partial R_{i} / \partial C_{j}, i \neq j\right)$ that are neglected by SIA-1 are significant. Physically, this can occur when aqueous, vapor, or immobile components that are, in fact, coupled to one another are treated as though they were independent.

\subsection{Selective coupling and coupled normalization}

Selective coupling: In an extension of the SIA methods, we selectively include additional derivative terms that couple a subset of the components to one another to improve convergence. We call this method "selective coupling" to denote the flexibility of the implementation. Since cross-derivative terms, $\partial R_{i} / \partial C_{j}$, are now included in the calculation, sets of components must be solved simultaneously. We note, in passing, that in the extreme case in which only $\partial R_{i} / \partial C_{i}$ are calculated, the method reduces to the SIA-1 method. At the other extreme, in which all components are coupled, a global implicit solution scheme [16] is effectively obtained. The algorithm is best illustrated through specific examples. For this, we first consider the following reaction system of kinetic reactions among aqueous components:

$$
\begin{aligned}
& A \Longleftrightarrow B ; \quad R_{\mathrm{A}}=k_{f_{1}} C_{\mathrm{A}}-k_{r_{1}} C_{\mathrm{B}}, \\
& B \Longleftrightarrow C ; \quad R_{\mathrm{B}}=k_{f_{2}} C_{\mathrm{B}}-k_{r_{2}} C_{\mathrm{C}}, \\
& C \Longleftrightarrow D ; \quad R_{\mathrm{C}}=k_{f_{3}} C_{\mathrm{C}}-k_{r_{3}} C_{\mathrm{D}} .
\end{aligned}
$$

We start with the fully coupled formulation of the transport problem. The equation set resulting from the use of Newton's method to solve the nonlinear system of equations is represented in block matrix form below

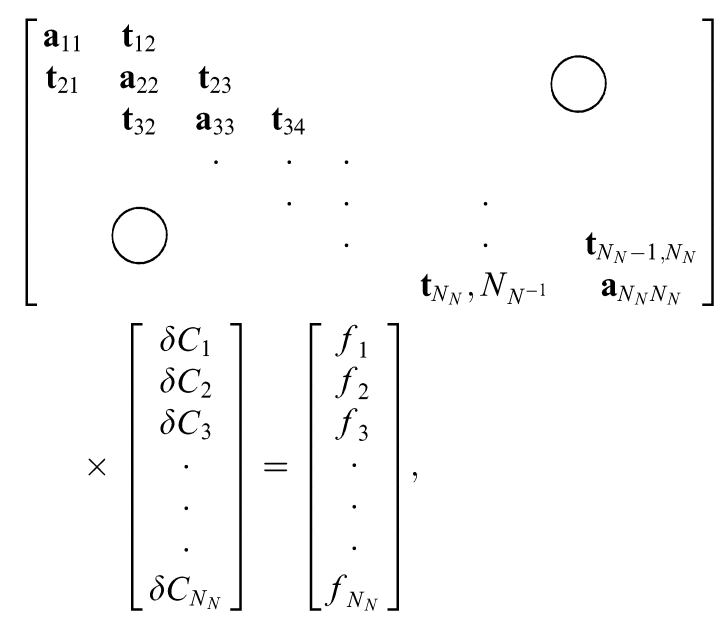

in which each element in the matrix is an $N_{\text {dof }} \times N_{\text {dof }}$ submatrix containing elements of the Jacobian matrix $\left(\partial f_{i} / \partial C_{j}\right), \delta C$ the vector of change in component concentration at node $i\left(\delta C_{\mathrm{A}}, \delta C_{\mathrm{B}}, \delta C_{\mathrm{C}}\right.$ and $\left.\delta C_{\mathrm{D}}\right), N_{\mathrm{N}}$ the number of spatial nodes, and $f_{i}$ are the residual arrays which contain the advection, dispersion, accumulation, and reaction terms (length of $N_{\text {dof }}$, where $N_{\text {dof }}$ is the number of "degrees of freedom", in this case equal to four, the number of components in the example). The elements of the Jacobian matrix contain derivatives of the residual with respect to concentration. In the diagram, a tri-diagonal matrix resulting from a one-dimensional transport problem is shown for simplicity, but is not a restriction of the method. For our example reactive transport system, the submatrix $\mathbf{a}$ and submatrix $\mathbf{t}$ at a node $q$ connected to node $q+1$ are given by

$\mathbf{a}=\left[\begin{array}{llll}\frac{\partial f_{\mathrm{A}}}{\partial C_{\mathrm{A}}} & \frac{\partial f_{\mathrm{A}}}{\partial C_{\mathrm{B}}} & & \bigcirc \\ \frac{\partial f_{\mathrm{B}}}{\partial \mathrm{C}_{\mathrm{A}}} & \frac{\partial f_{\mathrm{B}}}{\partial C_{\mathrm{B}}} & \frac{\partial f_{\mathrm{B}}}{\partial C_{\mathrm{C}}} & \\ & \frac{\partial f_{\mathrm{C}}}{\partial C_{\mathrm{B}}} & \frac{\partial f_{\mathrm{C}}}{\partial C_{\mathrm{C}}} & \frac{\partial f_{\mathrm{C}}}{\partial C_{\mathrm{D}}} \\ & & \frac{\partial f_{\mathrm{D}}}{\partial C_{\mathrm{C}}} & \frac{\partial f_{\mathrm{D}}}{\partial C_{\mathrm{D}}}\end{array}\right]$

$\mathbf{t}=\left[\begin{array}{ccc}\frac{\partial f_{\mathrm{A}}}{\partial C_{\mathrm{A}, q+1}} & & \bigcirc \\ & \frac{\partial f_{\mathrm{B}}}{\partial C_{\mathrm{B}, q+1}} & \\ & \frac{\partial f_{\mathrm{C}}}{\partial C_{C, q+1}} & \\ & & \frac{\partial f_{\mathrm{D}}}{\partial C_{D, q+1}}\end{array}\right], \quad q=1, \ldots, N_{N}$,

where the a matrix is written at node $q$, and $q+1$ represents a node connected to node $q$. In general, the structure of submatrix $\mathbf{a}$ is defined by the reaction system. Now suppose that reaction (23) is slow compared to the other two reactions. In the submatrix a, the terms 
$\partial f_{\mathrm{B}} / \partial C_{\mathrm{C}}$ and $\partial f_{\mathrm{C}} / \partial C_{\mathrm{B}}$ would be small compared to the other elements of $\mathbf{a}$, and a can be approximately represented as

$$
\mathbf{a}=\left[\begin{array}{cccc}
\frac{\partial f_{\mathrm{A}}}{\partial C_{\mathrm{A}}} & \frac{\partial f_{\mathrm{A}}}{\partial C_{\mathrm{B}}} & \\
\frac{\partial f_{\mathrm{B}}}{\partial C_{\mathrm{A}}} & \frac{\partial f_{\mathrm{B}}}{\partial C_{\mathrm{B}}} & & \\
\bigcirc & \frac{\partial f_{\mathrm{C}}}{\partial C_{\mathrm{C}}} & \frac{\partial f_{\mathrm{C}}}{\partial C_{\mathrm{D}}} \\
& \frac{\partial f_{\mathrm{D}}}{\partial C_{\mathrm{C}}} & \frac{\partial f_{\mathrm{D}}}{\partial C_{\mathrm{D}}}
\end{array}\right] .
$$

This system can then be solved in two steps, with the A and $\mathrm{B}$ equations solved simultaneously, followed by the $\mathrm{C}$ and $\mathrm{D}$ equations solved in a second step. A reactive transport computer code that employs this method allows the user to "selectively couple" the components in the transport iteration. The decomposition of a fourdegree-of-freedom problem into two-degrees-of-freedom problems results in memory efficiencies and computational savings per iteration, and therefore is a desirable alternative to a full global implicit solution for some applications. Note that when all off-diagonal terms in the submatrix a are omitted, each of the four components can be solved iteratively and sequentially, i.e. the SIA-1 method is obtained.

When more than one component is solved simultaneously, an equation solver that handles block matrices is required. The linear equation solver in FEHM, developed primarily for the solution of coupled fluid flow and heat transport, makes use of well-tested numerical techniques that take advantage of the block structure of the coupled equations for pressure, temperature, and fluid saturation [1,29]. Here, we use the same solver technologies for the transport solution step. In essence, for $N_{\text {dof }}$ unknowns per grid point, the same operations on the overall matrix of a single-unknown solution are carried out, but multiplications of individual matrix elements now become matrix multiplications involving the $N_{\text {dof }} \times N_{\text {dof }}$ submatrices, and divisions are carried out as multiplications by the inverse of the submatrix. Since such operations become memory- and cpu-intensive for large problems, it is important to employ efficient numerical techniques. FEHM uses numerical methods suitable for the nonsymmetric matrices that arise from the finite-element solution of reactive transport equations on unstructured numerical grids. The solver software uses incomplete factorization with variable fill-in level [30] as a preconditioner, and a generalized minimum residual (GMRES) acceleration technique [15] for the iterative solution. Details of this method as applied to heat and mass transport problems may be found in Zyvoloski et al. [31]. In a typical reactive transport solution with FEHM, the heat and mass transfer solution is also being performed, so the initial book-keeping associated with the method is already being carried out, and the memory allocated for the solution is shared between the heat and mass solution and the transport solution.

There are definite trade-offs in computational efficiency and memory utilization between the SIA techniques and methods involving coupling of the transport equations of individual components. Coupling requires more time per iteration and more memory than typical SIA methods. However, components strongly coupled by reaction may not converge using SIA methods without using small time steps. Since flexibility is required in a general purpose code, the transport iteration in FEHM was developed with the selective coupling provision to solve the component concentrations in groups of one or more at a time, so that only those components that need to be coupled are solved simultaneously. It is necessary to solve a set of equations for each component present in the system, but the order of solution and the nature of the coupling are set by the user at run-time. This allows the user, on the basis of information of the reactive transport system, to couple only those components that are required for efficient solution of the system of equations. Selective Coupling of components linked to each other through kinetic chemical reactions allows a given problem to be solved in the fastest, yet most memory efficient, manner possible [24].

Coupled normalization: When residual equations are solved simultaneously, it is advisable to normalize them so that they are solved to the same degree of numerical precision. We now present a method we call "coupled normalization" for accomplishing this in a manner that in some cases has the added benefit of effectively reducing the number of degrees of freedom of the solution. Again, we will make use of an example to illustrate the method, in this case a kinetic ion exchange reaction of the form

$\mathrm{A}+\mathrm{B}-\mathrm{X} \Longleftrightarrow \mathrm{B}+\mathrm{A}-\mathrm{X}$,
$R_{\mathrm{A}}=k_{\mathrm{f}}[\mathrm{A}][\mathrm{B}-\mathrm{X}]-k_{\mathrm{r}}[\mathrm{B}][\mathrm{A}-\mathrm{X}]$.

This example consists of two aqueous components (A, B), which in general can undergo aqueous speciation reactions (though not in this example) and two immobile components (A-X, B-X). As in the previous example, $N_{\text {dof }}$ is four because we will solve for these four components simultaneously. Furthermore, the block matrix equation set in (25) also applies here. The submatrices $\mathbf{a}$ and $\mathbf{t}$ are given by

$\mathbf{a}=\left[\begin{array}{cccc}\frac{\partial f_{\mathrm{A}}}{\partial C_{\mathrm{A}}} & \frac{\partial f_{\mathrm{A}}}{\partial C_{\mathrm{B}}} & \frac{\partial f_{\mathrm{A}}}{\partial M_{\mathrm{A}-\mathrm{X}}} & \frac{\partial f_{\mathrm{A}}}{\partial M_{\mathrm{B}-\mathrm{X}}} \\ \frac{\partial f_{\mathrm{B}}}{\partial C_{\mathrm{A}}} & \frac{\partial f_{\mathrm{B}}}{\partial C_{\mathrm{B}}} & \frac{\partial f_{\mathrm{B}}}{\partial M_{\mathrm{A}-\mathrm{X}}} & \frac{\partial f_{\mathrm{B}}}{\partial M_{\mathrm{B}-\mathrm{X}}} \\ \frac{\partial f_{\mathrm{A}-\mathrm{X}}}{\partial C_{\mathrm{A}}} & \frac{\partial f_{\mathrm{A}-\mathrm{X}}}{\partial C_{\mathrm{B}}} & \frac{\partial f_{\mathrm{A}-\mathrm{X}}}{\partial M_{\mathrm{A}-\mathrm{X}}} & \frac{\partial f_{\mathrm{A}-\mathrm{X}}}{\partial M_{\mathrm{B}-\mathrm{X}}} \\ \frac{\partial f_{\mathrm{B}-\mathrm{X}}}{\partial C_{\mathrm{A}}} & \frac{\partial f_{\mathrm{B}-\mathrm{X}}}{\partial C_{\mathrm{B}}} & \frac{\partial f_{\mathrm{B}-\mathrm{X}}}{\partial M_{\mathrm{A}-\mathrm{X}}} & \frac{\partial f_{\mathrm{B}-\mathrm{X}}}{\partial M_{\mathrm{B}-\mathrm{X}}}\end{array}\right]$ 
$\mathbf{t}=\left[\begin{array}{cc}\frac{\partial f_{\mathrm{A}}}{\partial C_{\mathrm{A}, q+1}} & \\ & \frac{\partial f_{\mathrm{B}}}{\partial C_{\mathrm{B}, q+1}} \\ & \end{array}\right]$.

The immobile components are not present in the $\mathbf{t}$ submatrices since there are no transport terms associated with them. Before solving the linear equation set, we apply the coupled normalization step, which consists of multiplying both sides of the equation set at each node by the inverse of a, which of course transforms each submatrix a into the identity matrix. This operation scales the diagonal term of each equation to the same value (unity), thereby normalizing the equation set to ensure that when a convergence criterion, such as the $L_{2}$ norm, is employed, each equation is solved to the same level of accuracy on a normalized basis. Coupled normalization serves another important function for systems with immobile components, as evidenced by the structure of the transport submatrix $\mathbf{t}^{\prime}\left(=\mathbf{t a}^{-\mathbf{1}}\right)$

$\mathbf{t}^{\prime}=\left[\begin{array}{llll}X & X & 0 & 0 \\ X & X & 0 & 0 \\ X & X & 0 & 0 \\ X & X & 0 & 0\end{array}\right]$

where the $X$ denotes a nonzero term. After coupled normalization, the mobile component equations no longer contain terms involving the immobile component unknowns in either the transformed submatrix a or the $\mathbf{t}^{\prime}$ submatrix. Thus, the first two equations can now be solved as a coupled two-by-two equation set for the concentration changes of the mobile species, after which the immobile component unknowns are then solved for individually by simple back-substitution. Coupled normalization in effect folds the cross-derivative information from the immobile component equations into the equations for the mobile components, so that only the two mobile component unknowns need be solved simultaneously. This method effectively reduces the number of degrees-of-freedom in the transport solution by the number of immobile components in the system of equations (two in this example), thereby reducing the memory utilization and computational burden of the solution.

\section{Test cases of the selective coupling technique}

The three examples in this section test the selective coupling technique and illustrate its power and flexibility for solving reactive transport problems. Example 1 is a hypothetical example in which six aqueous components transport and participate in kinetic reactions.
Example 1 illustrates the trade-offs involved in selecting the level of coupling and the influence that problem size and the nature of the chemical system will have on the techniques. We chose a simple flow system and a fictitious chemical system to gain insight into the controlling factors of the coupling strategy because other complexities tend to obscure these factors in real applications. In Example 2, we present a reactive transport problem in which we model the coupled effects of a set of equilibrium speciation reactions and kinetic biodegradation and adsorption/desorption reactions. Therefore, this problem builds on the results of Example 1, testing the methods with a realistic reactive transport system that includes solute-rock interactions and a mixed kinetic-equilibrium formulation. Finally, Example 3 shows how selective coupling performs for a field application, namely the potential repository for highlevel radioactive waste at Yucca Mountain, Nevada. The simulation utilizes an unstructured mesh with a large number of grid blocks and complex stratigraphy. This example illustrates the use of coupled normalization and selective coupling for significantly reducing the computational effort and memory requirements of the simulation. Thus, the lessons learned from Examples 1 and 2 provide the foundation for understanding the performance of the numerical techniques on a real field application.

Example 1. Kinetic formulation for reaction among aqueous components.

In this first example we investigate the solution options for aqueous components coupled through kinetic reactions. Although many aqueous speciation reactions can be specified as equilibrium, there are exceptions, such as microbially facilitated redox reactions [16]. Furthermore, in many applied reactive transport simulations, including those discussed in Examples 2 and 3 below, aqueous components undergo speciation reactions along with kinetic reactions such as competitive sorption. In these types of reactive systems, aqueous components often become tightly coupled through these kinetic reactions. Finally, even if the aqueous reactions are rapid, species with differing transport parameters such as dispersion or diffusion coefficients complicate the formulation of the system in terms of aqueous components and complexes (e.g. [10]). Alternatively, specifying aqueous components individually with different transport parameters is a way around this difficulty. This test case was developed to demonstrate the method of selective coupling with a reaction system in which all reactions are kinetic, even though for some reaction rates the problem could have been reformulated as an equilibrium reaction. The reaction system is a testbed for exploring the solution procedures that apply to more general reactive transport problems. 
The chemical system chosen for study is the following system of reactive aqueous components:

Reaction 1: $\mathrm{A}+\mathrm{B} \Longleftrightarrow \mathrm{C}$

Reaction $2: \mathrm{C} \Longleftrightarrow \mathrm{D}$

Reaction $3: \mathrm{D}+\mathrm{E} \Longleftrightarrow \mathrm{F}$

with flow conditions, initial and boundary conditions illustrated in the schematic diagram of Fig. 1. A onedimensional, steady-state flow system is used for this example, with initial concentrations of 0 (concentration units are arbitrary units) for all components except E, which initially is present everywhere in the column at concentration equal to 100 (or 0.1 for Cases 19-21). Components $\mathrm{A}$ and $\mathrm{B}$ are injected at a concentration of 1 for all times, and component $\mathrm{E}$ is injected at a concentration of 100 ( 0.1 for Cases 19-21). Rates of forward and reverse reactions are first order in all concentrations. For each reaction, the Damköhler number $\mathrm{Da}=k_{\mathrm{r}} \tau$, where $k_{\mathrm{r}}$ is the reverse rate constant and $\tau$ is the travel time through the column, is the dimensionless group that controls the relative rate of reaction, whereby $\mathrm{Da} \gg 1$ implies near-equilibrium behavior, and Da values of order 1 or less imply kinetically controlled reactions. In this exercise, we vary Da for the reactions to investigate the characteristics of the selective coupling method for obtaining convergent solutions with a minimum number of iterations.

In the first comparison (Cases 1-3), we assume $\mathrm{Da}=1$ for all three reactions. The selective coupling method is then applied (groups of components solved simultaneously are denoted by enclosing them in parentheses below) with the following options for the transport iteration:

- Case 1: All six components solved individually (SIA1), i.e. (A), (B), (C), (D), (E), and (F);

- Case 2: Coupling of components as $(\mathrm{A}, \mathrm{B}, \mathrm{C})$ followed by (D, E, F);

- Case 3: Coupling of all six components (A, B, C, D, E, F).

For all cases the convergence criterion is the same, and the iterative technique at each time step must achieve overall convergence before proceeding to the next time step. We treat the convergence criterion as a parameter whose value is governed by the need for an accurate

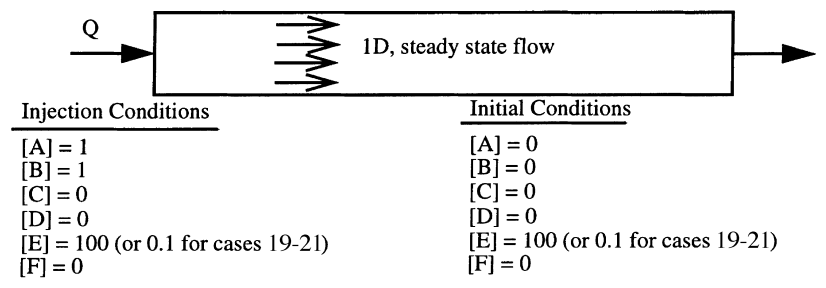

Fig. 1. Schematic of the one-dimensional model domain used for Example 1. solution, and therefore we do not examine the impact of its value on the performance of the algorithms. The simulations are run with a maximum Courant number of 1 (except for the explicit examples that test the influence of time step, Cases 10-12 and 13-15) until the breakthrough of each component at the outlet has achieved a steady concentration. In Table 1, a summary of the numerical performance for this comparison shows that the computational work associated with a particular iteration increases as the degree of coupling increases, but that fewer iterations are required to obtain a converged solution. However, for the case of $\mathrm{Da}=1$ for all three reactions, the uncoupled strategy yields somewhat better overall performance (lower total cpu time) than either coupling into 2 groups of 3 components or the fully coupled strategy. In fact, for this case with slow kinetics, operator splitting could be used with little mass balance error and should be even faster than the uncoupled strategy [23]. The benefits of the selective coupling method are better illustrated in Cases 4-6, a comparison assuming $\mathrm{Da}=100$ for the first and third reactions, and $\mathrm{Da}=1$ for the second reaction. Convergence becomes problematic for the SIA-1 method, and the resulting number of iterations and the total cpu time increase dramatically. However, the nature of the reaction system makes the intermediate level of coupling sufficient for this problem. The rapid kinetics of Reaction 1 makes coupling of $\mathrm{A}, \mathrm{B}$, and $\mathrm{C}$ necessary (the same is true for $\mathrm{D}, \mathrm{E}$, and $\mathrm{F}$ due to Reaction 3), but the breaking into these two groups is possible because Reaction 2 imparts only a relatively weak coupling of $\mathrm{A}, \mathrm{B}$, and $\mathrm{C}$ to $\mathrm{D}, \mathrm{E}$ and $\mathrm{F}$. When $\mathrm{Da}$ is increased to 1000 for the two reactions (Cases 79), the inadequacy of the uncoupled case becomes even more apparent.

To examine the influence of time step size on performance, Cases 10-12 and 13-15 were run with identical kinetic parameters as Cases $7-9$, but with a maximum Courant number of 0.2 (Cases 10-12) or 5 (Cases 13-15). Cases 10-12 show that, as expected, lowering the time step makes the uncoupled case somewhat more competitive. However, the usefulness of coupling the aqueous components is best illustrated in Cases 13-15, in which convergence cannot even be obtained without coupling. Thus the coupling strategy allows larger time steps to be taken, whereas numerical stability becomes an issue without coupling.

In Cases $16-18, \mathrm{Da}$ is increased to 1000 for Reaction 2. At this point, the fully coupled strategy begins to slightly outperform the intermediate coupled strategy, as the reduction in the number of iterations required is enough to overcome the greater cpu time per iteration. Increasing the rate of Reaction 2 imparts a greater degree of coupling between the two sets of components, thereby causing the performance of the intermediate coupling strategy to begin to deteriorate. 
Table 1

Computational efficiency for different levels of selective coupling: Example 1

\begin{tabular}{|c|c|c|c|c|c|}
\hline Case number & Coupling & $\begin{array}{l}\text { Damköhler numbers for the } \\
\text { three reactions }\left(\mathrm{Da}_{1}, \mathrm{Da}_{2}, \mathrm{Da}_{3}\right)\end{array}$ & $\begin{array}{l}\text { Total number } \\
\text { of iterations }\end{array}$ & $\begin{array}{l}\text { cpu time per } \\
\text { iteration }(\mathrm{s})\end{array}$ & $\begin{array}{l}\text { Total cpu } \\
\text { time }(\mathrm{s})\end{array}$ \\
\hline 1 & $(\mathrm{~A})(\mathrm{B})(\mathrm{C})(\mathrm{D})(\mathrm{E})(\mathrm{F})$ & $(1,1,1)$ & 2915 & 0.073 & 212 \\
\hline 2 & $(\mathrm{~A} B \mathrm{C})(\mathrm{DEF})$ & $(1,1,1)$ & 2801 & 0.091 & 254 \\
\hline 3 & (A B C D E F) & $(1,1,1)$ & 2206 & 0.14 & 312 \\
\hline 4 & $(\mathrm{~A})(\mathrm{B})(\mathrm{C})(\mathrm{D})(\mathrm{E})(\mathrm{F})$ & $(100,1,100)$ & 6876 & 0.073 & 501 \\
\hline 5 & $(\mathrm{~A} B \mathrm{C})(\mathrm{DEF})$ & $(100,1,100)$ & 2825 & 0.086 & 243 \\
\hline 6 & $(\mathrm{~A} B \mathrm{C} D \mathrm{EF})$ & $(100,1,100)$ & 2681 & 0.14 & 379 \\
\hline 7 & (A) (B) (C) (D) (E) (F) & $(1000,1,1000)$ & 80,982 & 0.071 & 5752 \\
\hline 8 & $(\mathrm{~A} B \mathrm{C})(\mathrm{DEF})$ & $(1000,1,1000)$ & 2836 & 0.087 & 246 \\
\hline 9 & $(\mathrm{~A} B \mathrm{C} D \mathrm{EF})$ & $(1000,1,1000)$ & 2756 & 0.14 & 387 \\
\hline $10^{\mathrm{a}}$ & (A) (B) (C) (D) (E) (F) & $(1000,1,1000)$ & 36,825 & 0.078 & 2868 \\
\hline $11^{\mathrm{a}}$ & $(\mathrm{A} B \mathrm{C})(\mathrm{DEF})$ & $(1000,1,1000)$ & 10,500 & 0.094 & 985 \\
\hline $12^{\mathrm{a}}$ & (A B C D E F) & $(1000,1,1000)$ & 10,488 & 0.15 & 1524 \\
\hline $13^{\mathrm{b}}$ & $(\mathrm{A})(\mathrm{B})(\mathrm{C})(\mathrm{D})(\mathrm{E})(\mathrm{F})$ & $(1000,1,1000)$ & No convergence & N/A & N/A \\
\hline $14^{\mathrm{b}}$ & $(\mathrm{A} B \mathrm{C})(\mathrm{DEF})$ & $(1000,1,1000)$ & 861 & 0.093 & 80 \\
\hline $15^{\mathrm{b}}$ & (A B C D E F) & $(1000,1,1000)$ & 744 & 0.14 & 104 \\
\hline 16 & $(\mathrm{~A})(\mathrm{B})(\mathrm{C})(\mathrm{D})(\mathrm{E})(\mathrm{F})$ & $(1000,1000,1000)$ & 36,792 & 0.073 & 2691 \\
\hline 17 & $(\mathrm{~A} B \mathrm{C})(\mathrm{D} E \mathrm{~F})$ & $(1000,1000,1000)$ & 4064 & 0.086 & 350 \\
\hline 18 & $(\mathrm{~A} B \mathrm{C} D \mathrm{EF})$ & $(1000,1000,1000)$ & 2313 & 0.14 & 328 \\
\hline $19^{c}$ & $(\mathrm{~A})(\mathrm{B})(\mathrm{C})(\mathrm{D})(\mathrm{E})(\mathrm{F})$ & $(1000,1000,1000)$ & 76,863 & 0.073 & 5557 \\
\hline $20^{c}$ & $(\mathrm{~A} B \mathrm{C})(\mathrm{DEF})$ & $(1000,1000,1000)$ & 11,058 & 0.083 & 915 \\
\hline $21^{\mathrm{c}}$ & $(\mathrm{A} B \mathrm{C} D \mathrm{E} F)$ & $(1000,1000,1000)$ & 2766 & 0.14 & 389 \\
\hline
\end{tabular}

${ }^{a}$ Same as Cases 7-9, except that the Courant number is 0.2 .

${ }^{\mathrm{b}}$ Same as Cases 7-9, except that the Courant number is 5 .

${ }^{\mathrm{c}}$ Initial and injection concentrations of $\mathrm{E}$ are 0.1 rather than 100 for the previous cases.

The final comparison (Cases 19-21) illustrates that performance deteriorates markedly for even the intermediate degree of coupling when the injection and initial concentrations of $\mathrm{E}$ are reduced from 100 to 0.1. Although it is still possible to obtain a solution at the intermediate level of coupling, the number of iterations required and the total cpu time increase markedly for the uncoupled and intermediate coupling strategies. For concentration of $\mathrm{E}$ of 100 , the forward reaction of Reaction 3 is essentially pseudo-first order in D. Reducing the concentration of $\mathrm{F}$ to 0.1 changes the character of Reaction 3, making the concentrations of A, B and C much more closely linked to the concentrations of D, E and $\mathrm{F}$. These results illustrate that the proper strategy for selective coupling from the standpoint of computational efficiency depends on the nature of the chemical system, including the stoichiometry and kinetics of the reactions and the concentration values themselves.

For large-scale simulations, the memory required to obtain a solution, rather than the cpu time, may constrain the choice of solution strategy. For the selective coupling method, memory requirements can be severe for large simulations if many components need to be coupled to obtain a solution efficiently. We use this reaction system as an example to illustrate this point for a hypothetical three-dimensional grid consisting of $N_{\text {cell }}$ cells. The Jacobian matrix requires a storage of $N_{\text {dof }}^{2} N_{\text {cell }}$ for the a submatrices in Eq. (26), and approximately $N_{\text {dof }} N_{\text {con }} N_{\text {cell }}$ for the $\mathbf{t}$ submatrices, or a total of $N_{\text {dof }}\left(N_{\text {dof }}+N_{\text {con }}\right) N_{\text {cell }}$, where $N_{\text {con }}$ is the average number of neighboring nodes connected to each node. In addition, incomplete factorization methods require the storage of a partial inversion of the Jacobian matrix in which, at a minimum, the $\mathbf{t}$ submatrices become filled in, for a total storage of $N_{\text {dof }}^{2}\left(N_{\text {con }}+1\right) N_{\text {cell }}$. The $N_{\text {dof }}^{2}$ dependence of the storage places a premium on memory for simulations with a large number of grid points and high degrees of coupling. Table 2 shows the memory requirements for a specific example of a 100,000 node, hexahedral grid $\left(N_{\text {cell }}=100,000, N_{\text {con }}=6\right)$, for the various degrees of coupling studied in this reaction system. Furthermore, for more than one level of fill-in for the incomplete factorization, storage requirements are even more severe for the inversion matrix. Therefore, in largescale simulations, memory requirements alone mandate that selective coupling be applied such that only the minimum degree of coupling needed for efficient convergence is used. For smaller problems, the decision of the degree of coupling hinges only on the cpu time issue, making the more robust full coupling of the solution an

Table 2

Memory efficiency for a hypothetical 100,000 node simulation for different levels of selective coupling

\begin{tabular}{llll}
\hline Coupling & $\begin{array}{l}\text { Maximum } \\
N_{\text {dof }}\end{array}$ & $\begin{array}{l}\text { Size of } \\
\text { Jacobian } \\
\text { (words) }\end{array}$ & $\begin{array}{l}\text { Minimum size of } \\
\text { partial inversion of } \\
\text { Jacobian (words) }\end{array}$ \\
\hline (A) (B) (C) (D) (E) (F) & 1 & $7.0 \times 10^{5}$ & $7.0 \times 10^{5}$ \\
(A B C) (D E F) & 3 & $2.7 \times 10^{6}$ & $6.3 \times 10^{6}$ \\
(A B C D E F) & 6 & $7.2 \times 10^{6}$ & $2.5 \times 10^{7}$ \\
\hline
\end{tabular}


attractive alternative. The optimal use of computer resources is therefore dependent on the size of $N_{\text {cell }}$, and the nature of the chemical system, which in turn controls the efficiency of the numerical solution. Allowing the selection of partial levels of coupling allows the user to tailor the solution technique to the specific application.

\section{Example 2. CoNTA Transport Problem.}

In the next example, we model the transport of ${ }^{60} \mathrm{Co}$ in the presence of inorganic and organic chemical waste. We depart from the theoretical nature of Example 1 to explore a more realistic reactive transport problem. The objective of the analysis is to model the coupled effects of a set of equilibrium speciation reactions and kinetic biodegradation and adsorption/desorption reactions. This problem was proposed by Valocchi and TebesStevens as a benchmark problem at the Workshop on Subsurface Reactive Transport Modeling held at Pacific Northwest National Laboratory (October, 1997). The reactions are described in more detail by Tebes-Stevens and Valocchi [20]. This reactive transport system has practical significance since soils and groundwater at DOE facilities have been contaminated by complex mixtures of radioactive, inorganic, and organic chemical wastes. In particular, Cobalt, as ${ }^{60} \mathrm{Co}$, is a radioactive contaminant that has been found migrating in the subsurface at several DOE facilities [20]. The mobility of ${ }^{60} \mathrm{Co}$ has been greater than anticipated due to complexation with organic ligands such as EDTA and NTA [20].

A one-dimensional column 10 meters in length was chosen for this simulation. The porosity, $\phi$, is 0.4 , the bulk rock density, $\rho_{\mathrm{b}}$ is $1.5 \times 10^{3} \mathrm{~kg} / \mathrm{m}^{3}$, the pore water velocity is $1 \mathrm{~m} / \mathrm{h}$, and the longitudinal dispersivity is 0.05 $\mathrm{m}$. Table 3 lists the components and concentrations of the background and injected fluid, while Table 4 shows the equilibrium speciation reactions.

The biodegradation of the complex HNTA $^{2-}$ is represented by the following reaction [20]:

$$
\begin{aligned}
& \mathrm{HNTA}^{2-}+1.620 \mathrm{O}_{2}+1.272 \mathrm{H}_{2} \mathrm{O}+2.424 \mathrm{H}^{+} \\
& \quad \rightarrow 0.576 \mathrm{C}_{5} \mathrm{H}_{7} \mathrm{O}_{2} \mathrm{~N}+3.120 \mathrm{H}_{2} \mathrm{CO}_{3}+0.424 \mathrm{NH}_{4}^{+} .
\end{aligned}
$$

Table 4

\begin{tabular}{|c|c|}
\hline Reaction & $\log K_{\mathrm{eq}}$ \\
\hline $\mathrm{H}_{3} \mathrm{NTA} \Longleftrightarrow \mathrm{NTA}^{3-}+3 \mathrm{H}^{+}$ & 14.9 \\
\hline $\mathrm{H}_{2} \mathrm{NTA}^{-} \Longleftrightarrow \mathrm{NTA}^{3-}+2 \mathrm{H}^{+}$ & 13.3 \\
\hline $\mathrm{HNTA}^{2-} \Longleftrightarrow \mathrm{NTA}^{3-}+\mathrm{H}^{+}$ & 10.3 \\
\hline $\mathrm{H}_{2} \mathrm{O} \Longleftrightarrow \mathrm{OH}^{-}+\mathrm{H}^{+}$ & 14.0 \\
\hline $\mathrm{CoNTA}^{-} \Longleftrightarrow \mathrm{NTA}^{3-}+\mathrm{Co}^{2+}$ & 11.7 \\
\hline $\mathrm{CoNTA}_{2}^{4-} \Longleftrightarrow 2 \mathrm{NTA}^{3-}+\mathrm{Co}^{2+}$ & 14.5 \\
\hline $\mathrm{CoOHNTA}{ }^{2-} \Longleftrightarrow \mathrm{NTA}^{3-}+\mathrm{Co}^{2+}+\mathrm{OH}^{-}$ & 0.5 \\
\hline $\mathrm{CoOH}^{+} \Longleftrightarrow \mathrm{Co}^{2+}+\mathrm{OH}^{-}$ & -9.7 \\
\hline $\mathrm{Co}(\mathrm{OH})_{2} \Longleftrightarrow \mathrm{Co}^{2+}+2 \mathrm{OH}^{-}$ & -22.9 \\
\hline $\mathrm{Co}(\mathrm{OH})_{3}^{-} \Longleftrightarrow \mathrm{Co}^{2+}+2 \mathrm{OH}^{-}$ & -31.5 \\
\hline $\mathrm{HCO}_{3}^{-}+\mathrm{H}^{+} \Longleftrightarrow \mathrm{H}_{2} \mathrm{CO}_{3}^{*}$ & -6.35 \\
\hline $\mathrm{CO}_{3}^{2-}+2 \mathrm{H}^{+} \Longleftrightarrow \mathrm{H}_{2} \mathrm{CO}_{3}^{*}$ & -16.68 \\
\hline $\mathrm{NH}_{3}+\mathrm{H}^{+} \Longleftrightarrow \mathrm{NH}_{4}^{+}$ & -9.3 \\
\hline
\end{tabular}

Equilibrium speciation reactions, CoNTA transport problem

The rate of substrate degradation $\left(R_{\mathrm{HNTA}}\right)$ is modeled with multiplicative-Monod kinetics given by Eq. (12). The parameters used in Eq. (12) are: $K_{\mathrm{S}}=7.64 \times 10^{-7} \mathrm{~mol} / 1, \quad K_{\mathrm{A}}=6.25 \times 10^{-6} \mathrm{~mol} / 1$, and $q_{\mathrm{m}}=1.407 \times 10^{-3} \mathrm{~mol} / \mathrm{NTA} / \mathrm{g}$ cells $/ \mathrm{h}$ [20]. The reaction rates for $\mathrm{O}_{2}, \mathrm{H}^{+}, \mathrm{H}_{2} \mathrm{CO}_{3}$ and $\mathrm{NH}_{4}^{+}$are proportional to the rate of substrate degradation. Using the stoichiometry of the above reaction, the appropriate rate expressions are:

$$
\begin{aligned}
& R_{\mathrm{O}_{2}}=1.602 R_{\mathrm{HNTA}^{2-}}, \quad R_{\mathrm{H}^{+}}=2.424 R_{\mathrm{HNTA}^{2-}}, \\
& R_{\mathrm{H}_{2} \mathrm{CO}_{3}}=-3.120 R_{\mathrm{HNTA}^{2-}}, \quad R_{\mathrm{NH}_{4}^{+}}=1.620 R_{\mathrm{HNTA}^{2-}}
\end{aligned}
$$

The net rate of microbial growth $\left(R_{\text {cells }}\right)$ is given by the synthesis rate (which is equal to the rate of degradation of the substrate multiplied by a yield coefficient) minus a first-order decay rate:

$\mathrm{R}_{\text {cells }}=-\mathrm{YR}_{\mathrm{HNTA}^{2-}}-b \mathrm{X}_{m}$,

where $Y$ is the yield coefficient and $b$ is the decay coefficient. The parameters $Y$ and $b$ are $65.15 \mathrm{~g}$ cells/mole NTA and $0.00208 \mathrm{~h}^{-1}$, respectively.

The sorption reactions are represented by a linear kinetic model given by (10). In this problem, uncomplexed cobalt $\left(\mathrm{Co}^{2+}\right)$ and $\mathrm{CoNTA}^{-}$are retarded due to sorption. The distribution coefficients, $K_{\mathrm{d}}$, for $\mathrm{Co}^{2+}$ and CoNTA $^{-}$are assumed to be equal to $5.07 \times 10^{-3} \mathrm{~m}^{3} / \mathrm{g}$ and $5.33 \times 10^{-4} \mathrm{~m}^{3} / \mathrm{g}$, respectively. Note that these

Table 3

Components and concentrations of the background and injected fluid, CoNTA transport problem

\begin{tabular}{llll}
\hline Component & Type & Pulse concentration & Background concentration \\
\hline $\mathrm{H}^{+}$ & Aqueous & $\mathrm{pH}=6$ & $\mathrm{pH}=6$ \\
$\mathrm{H}_{2} \mathrm{CO}_{3}$ & Aqueous & $4.9 \times 10^{-7} \mathrm{~mol} / \mathrm{l}$ & $4.9 \times 10^{-7} \mathrm{~mol} / 1$ \\
$\mathrm{NH}_{4}^{+}$ & Aqueous & 0.0 & 0.0 \\
$\mathrm{O}_{2}$ & Aqueous & $3.125 \times 10^{-5} \mathrm{~mol} / \mathrm{l}$ & $3.125 \times 10^{-5} \mathrm{~mol} / 1$ \\
$\mathrm{NTA}^{3-}$ & Aqueous & $5.23 \times 10^{-6} \mathrm{~mol} / \mathrm{l}$ & 0.0 \\
$\mathrm{Co}^{2+}$ & Aqueous & $5.23 \times 10^{-6} \mathrm{~mol} / \mathrm{l}$ & 0.0 \\
Biomass & Immobile & - & $1.36 \times 10^{-4} \mathrm{~g} / 1$ \\
CoNTA(ads) & Immobile & - & 0.0 \\
Co(ads) & Immobile & - & 0.0 \\
\hline
\end{tabular}


distribution coefficients were selected to give retardation coefficients of 20 and 3 for $\mathrm{Co}^{2+}$ and CoNTA, respectively. The $K_{\mathrm{d}}$ values are chosen for demonstration of the numerical techniques, and do not necessarily represent realistic sorption behavior.

This test problem was broken up into two parts. In the first part of this problem, part A, the mass transfer coefficients for the sorption reactions, were set to $1 \mathrm{~h}^{-1}$. In part $\mathrm{B}$, the mass transfer coefficient is increased to $1000 \mathrm{~h}^{-1}$. Setting the mass transfer coefficient to 1000 $\mathrm{h}^{-1}$ approximates equilibrium for the sorption reactions, resulting in sharper concentration fronts. Fig. 2 shows that the FEHM solution closely matches the solution presented by Valocchi and Tebes-Stevens [20] for part A. Note that Cases 1, 2 and 3 all result in accurate solutions to the problem, with the difference in the techniques being computational efficiency. Similar agreement is witnessed for part B.

As with the first example problem, the selective coupling method is then applied with the following options for the transport iteration to solve this problem

- Case 1: All six aqueous components solved individually (SIA-1), i.e. $\left(\mathrm{H}^{+}\right),\left(\mathrm{H}_{2} \mathrm{CO}_{3}\right),\left(\mathrm{NH}_{4}^{+}\right),\left(\mathrm{O}_{2}\right)$, $\left(\mathrm{NTA}^{3-}\right)$, and $\left(\mathrm{Co}^{2+}\right)$. Biomass, CoNTA(ads) and Co(ads) solid components are solved individually;

- Case 2: Coupling of aqueous components $\left(\mathrm{Co}^{2+}\right)$ and $\left(\mathrm{NTA}^{3-}\right)$ followed by $\left(\mathrm{H}^{+}\right),\left(\mathrm{H}_{2} \mathrm{CO}_{3}\right),\left(\mathrm{NH}_{4}^{+}\right)$, and $\left(\mathrm{O}_{2}\right)$, which are solved individually. Biomass, CoN-



Fig. 2. Comparison of FEHM (full circles) and the Tebes-Stevens and Valocchi ${ }^{20}$ (lines) solution for part A.
TA(ads) and Co(ads) solid components are solved individually;

- Case 3: Coupling of all six aqueous components $\left(\mathrm{H}^{+}\right)$, $\left(\mathrm{H}_{2} \mathrm{CO}_{3}\right),\left(\mathrm{NH}_{4}^{+}\right),\left(\mathrm{O}_{2}\right),\left(\mathrm{NTA}^{3-}\right)$, and $\left(\mathrm{Co}^{2+}\right)$ (global implicit method). Biomass, CoNTA(ads) and Co(ads) solid components are solved individually.

Recall that the reaction rates for Co and NTA are coupled to one another due to the competitive sorption reactions whereas the other aqueous components are not strongly coupled to one another due to kinetic reactions. Thus, we chose to couple only cobalt and NTA in Case 2. Table 5 compares the three cases. For part A, Case 1 and Case 2 are comparable in total CPU time, whereas Case 3 takes longer to run. In part $\mathrm{A}$, the $\partial R_{i} / \partial C_{j}$ are small due to the slow sorption kinetic parameters. Therefore, Case 1 performs as well as Case 2. Since SIA1 (Case 1) and selective coupling of cobalt and NTA (Case 2) solve smaller equation sets than the global implicit method (Case 3), these methods are more efficient. For this problem, SIA-1 method solves six $N_{\mathrm{c}} \times N_{\mathrm{c}}$ matrices. Selective coupling solves one $2 N_{\mathrm{c}} \times 2 N_{\mathrm{c}}$ matrix (for Co and NTA), and four $N_{\mathrm{c}} \times N_{\mathrm{c}}$ matrices for the remaining aqueous components. The global implicit method solves one $6 N_{\mathrm{c}} \times 6 N_{\mathrm{c}}$ matrix solving for all the aqueous components simultaneously. All three methods solve for the solids individually. In part B, the coupling between Co and NTA is more important due to the faster kinetic rates. SIA-1 requires, on average, 7.5 iterations per time step whereas selective coupling and the global implicit methods require only 3.5 iterations per time step. Although the time for each iteration is still faster for SIA-1 than for the other two methods, more iterations per time step are required for convergence for SIA-1 resulting in larger run times. The SIA-1 method requires a large number of iterations per time step to converge since the cross derivative terms $\left(\Delta t \partial R_{i} / \partial C_{j}\right)$ neglected by SIA-1 are now significant in part B. Selective coupling outperforms the global implicit method since it includes the necessary cross-derivative terms, but still neglects most of the other insignificant cross-derivative terms computed by the global implicit solution.

Example 3. ${ }^{237} \mathrm{~Np}$ Reactive Transport at Yucca Mountain.

In this example, we simulate the unsaturated zone transport of ${ }^{237} \mathrm{~Np}$ from the potential high-level waste repository at Yucca Mountain, Nevada. For a detailed description of the hydrologic and geochemical processes affecting the migration of ${ }^{237} \mathrm{~Np}$, see Viswanathan et al. [25]. This problem demonstrates the selective coupling method for a complex, unstructured grid and a reactive transport system with speciation and competitive ion exchange. By choosing a model with a relatively large and complex grid, and mixed equilibrium-kinetic chemical formulation, we examine the numerical per- 
Table 5

Computational efficiency, CoNTA transport problem

\begin{tabular}{|c|c|c|c|c|c|c|}
\hline \multirow[t]{2}{*}{ Problem set } & \multicolumn{2}{|l|}{ Case 1 - SIA-1 } & \multicolumn{2}{|c|}{$\begin{array}{l}\text { Case } 2-\text { Selective coupling: } \\
\mathrm{Co}^{2+} \text { and } \mathrm{NTA}^{3-}\end{array}$} & \multicolumn{2}{|c|}{ Case 3 - Global implicit method } \\
\hline & $\begin{array}{l}\text { Average no. of } \\
\text { iterations per } \\
\text { time step }\end{array}$ & $\begin{array}{l}\text { Total CPU } \\
\text { time (s) }\end{array}$ & $\begin{array}{l}\text { Average no. of } \\
\text { iterations per } \\
\text { time step }\end{array}$ & $\begin{array}{l}\text { Total CPU } \\
\text { time (s) }\end{array}$ & $\begin{array}{l}\text { Average no. of } \\
\text { iterations per } \\
\text { time step }\end{array}$ & $\begin{array}{l}\text { Total CPU } \\
\text { time (s) }\end{array}$ \\
\hline Part A & 3.0 & 339 & 2.8 & 320 & 2.8 & 436 \\
\hline Part B & 7.5 & 888 & 3.5 & 417 & 3.5 & 525 \\
\hline
\end{tabular}

formance on a system for which the model was developed, namely large field applications. The ion exchange reactions require that solid components be coupled with aqueous components. Therefore, this problem also demonstrates the efficiency of coupled normalization. For the purposes of the current study we will briefly summarize the problem and then demonstrate the performance of the method.

The domain selected for the transport calculations is a two-dimensional, East-West cross section through Yucca Mountain at the location of the potential repository. Fig. 3(a) shows the East-dipping stratigraphy at this cross section, including the zeolitic horizons important to the transport of neptunium. The finite element grid for the entire cross section is shown in Fig. 3 (b) and (c). The unstructured grid captures the complex stratigraphy at the site scale, while also allowing transport near the potential repository to be captured at a grid spacing of about $3 \mathrm{~m}$, resulting in a mesh with 7070 spatial nodes. For details on the hydrologic models, parameters, and infiltration fluxes used to investigate ${ }^{237} \mathrm{~Np}$ transport, see Viswanathan et al. [25]. In the present study, the nonisothermal effects examined in that paper are ignored in favor of an isothermal model, so that the chemical transport processes and numerical schemes can be examined more directly.

The geochemical processes that strongly affect ${ }^{237} \mathrm{~Np}$ migration include: solubility-limited release of ${ }^{237} \mathrm{~Np}$ from the repository, aqueous speciation of neptunium into non-sorbing carbonate/hydroxy complexes and the sorbing $\mathrm{NpO}_{2}^{+}$cation, sorption of ${ }^{237} \mathrm{~Np}$ onto the zeolitic tuffs via an ion exchange mechanism, and radioactive decay. Solubilities for groundwater representative of Yucca Mountain were obtained from Efurd et al. [5]. We model the precipitation-dissolution of neptunium at the repository using Eq. (16), with the kinetic parameter


Fig. 3. Stratigraphy and numerical grid for the Neptunium reactive transport problem (Example 3). (a) Dipping stratigraphy, including the location of zeolitic horizons. (b) Numerical grid of full model domain. (c) Close-up of grid at the edge of the potential repository. 
value chosen to ensure that the reaction proceeds to equilibrium. The aqueous speciation reactions for Neptunium are given in Table 6 . The three total aqueous components chosen to model these reactions are $\mathrm{NpO}_{2}^{+}, \mathrm{HCO}_{3}^{-}$, and $\mathrm{H}^{+}$.

To simulate the sorption of $\mathrm{NpO}_{2}^{+}$cation, we couple the speciation results presented in Table 6 with an ion exchange model for $\mathrm{NpO}_{2}^{+}$. The following set of ion exchange reactions are assumed in the present study

$$
\begin{aligned}
& \mathrm{NpO}_{2}^{+}+\mathrm{tA} 1-\mathrm{Na}^{+} \Longleftrightarrow \mathrm{tA} 1-\mathrm{NpO}_{2}^{+}+\mathrm{Na}^{+}, \\
& \log _{10} K=-1.58, \\
& \mathrm{Ca}^{2+}+2 \mathrm{tAl}-\mathrm{Na}^{+} \Longleftrightarrow(2 \mathrm{tA} 1)-\mathrm{Ca}^{2+}+2 \mathrm{Na}^{+}, \\
& \log _{10} K=1.5,
\end{aligned}
$$

where tA1 represents a tetrahedral aluminum sorption site [25]. We model these reactions with kinetic rate laws. However, since appropriate values of the rate constant are uncertain, we examine two kinetic regimes differing by an order of magnitude in the rate constant. The main chemical influences on the extent of sorption onto the zeolitic tuffs according to this model are $\mathrm{pH}$ (controlled by the speciation reactions) and the concentrations of the competing cations in the sorption reactions, $\mathrm{Na}^{+}$ and $\mathrm{Ca}^{2+}$. This problem consists of five total aqueous components: $\mathrm{NpO}_{2}^{+}, \mathrm{HCO}_{3}^{-}, \mathrm{H}^{+}, \mathrm{Na}^{+}$, and $\mathrm{Ca}^{2+}$, and four immobile components: $\mathrm{Np}(\mathrm{s}), \quad \mathrm{tA} 1-\mathrm{NpO}_{2}^{+}$, $(2 \mathrm{tA} 1)-\mathrm{Ca}^{2+}, \mathrm{tA} 1-\mathrm{Na}^{+}$.

Fig. 4 shows the mass flux breakthrough at the water table of Neptunium for a solution of $\mathrm{pH}=8$, $\left[\mathrm{Na}^{+}\right]=5.43 \times 10^{-3} \mathrm{M}$, and $\left[\mathrm{Ca}^{2+}\right]=2.91 \times 10^{-4} \mathrm{M}$ assuming equilibrium sorption (Curve a). For reference, the breakthrough of a solute with the same release concentration but no sorption on the zeolites is also shown (Curve d). Retardation in the zeolitic tuffs is an important process for predicting the migration of Neptunium through the unsaturated zone. With regard to the solution technique, this example illustrates the use of the coupled normalization step for properly linking the aqueous and immobile species in the numerical solution.

Table 6

Equilibrium speciation reactions, Neptunium transport problem

\begin{tabular}{lc}
\hline Reaction & $\log K_{\mathrm{eq}}$ \\
\hline $\mathrm{H}_{2} \mathrm{CO}_{3}(\mathrm{aq}) \Longleftrightarrow \mathrm{HCO}_{3}^{-}+\mathrm{H}^{+}$ & -6.34 \\
$\mathrm{HCO}_{3}^{-} \Longleftrightarrow \mathrm{CO}_{3}^{2-}+\mathrm{H}^{+}$ & 10.33 \\
$\mathrm{H}_{2} \mathrm{O} \Longleftrightarrow \mathrm{OH}^{-}+\mathrm{H}^{+}$ & -14.17 \\
$\mathrm{NpO}_{2}^{+}+\mathrm{H}_{2} \mathrm{O} \Longleftrightarrow \mathrm{NpO}_{2}(\mathrm{OH})^{0}(\mathrm{aq})+\mathrm{H}^{+}$ & 8.9 \\
$\mathrm{NpO}_{2}^{+}+2 \mathrm{H}_{2} \mathrm{O} \Longleftrightarrow \mathrm{NpO}_{2}(\mathrm{OH})_{2}^{-}+2 \mathrm{H}^{+}$ & 20.2 \\
$\mathrm{NpO}_{2}^{+}+\mathrm{HCO}_{3}^{-} \Longleftrightarrow \mathrm{NpO}_{2}\left(\mathrm{CO}_{3}\right)^{-}+\mathrm{H}^{+}$ & 5.73 \\
$\mathrm{NpO}_{2}^{+}+2\left(\mathrm{HCO}_{3}\right)^{-} \Longleftrightarrow \mathrm{NpO}_{2}\left(\mathrm{CO}_{3}\right)_{2}^{3-}+2 \mathrm{H}^{+}$ & 13.66 \\
$\mathrm{NpO}_{2}^{+}+3\left(\mathrm{HCO}_{3}^{-}\right) \Longleftrightarrow \mathrm{NpO}_{2}\left(\mathrm{CO}_{3}\right)_{3}^{5-}+3 \mathrm{H}^{+}$ & 22.49 \\
\hline
\end{tabular}

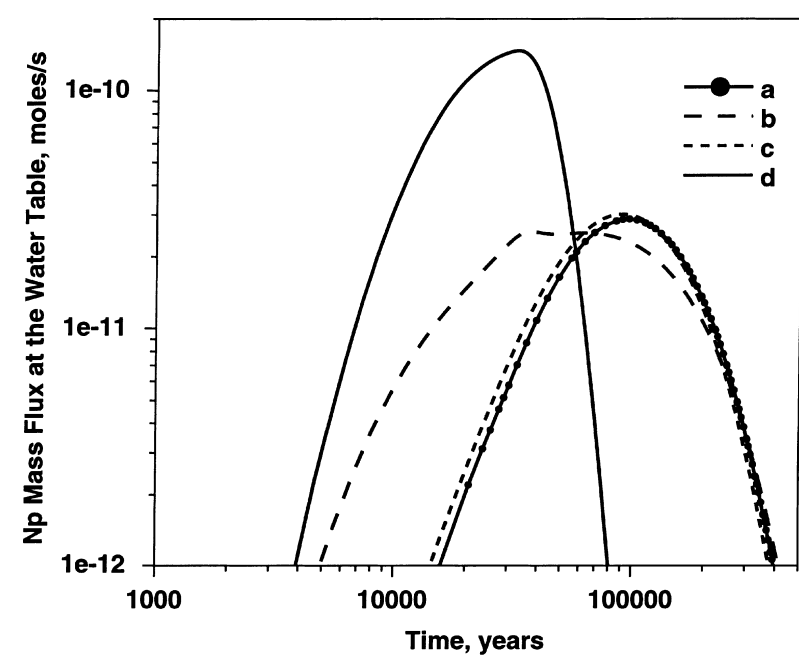

Fig. 4. Neptunium breakthrough mass flux at the water table. Curve (a) equilibrium sorption reactions. (b) Slow reaction kinetics for sorption reactions (Case 1). (c) Rapid reaction kinetics for sorption reactions (Case 2). (d) Conservative tracer with the same repository release rate as (a)-(c).

To demonstrate this, we performed a series of simulations with different coupling strategies, examining the relative performance for two different reaction rates

- Case 1a: The components $\mathrm{HCO}_{3}^{-}, \mathrm{Np}(\mathrm{s})$ and $\mathrm{H}^{+}$were solved for individually. The components $\mathrm{NpO}_{2}^{+}, \mathrm{Ca}^{2+}, \mathrm{Na}^{+}, \mathrm{tA} 1-\mathrm{NpO}_{2}^{+},(2 \mathrm{tA} 1)-\mathrm{Ca}^{2+}, \quad$ and tA1-Na ${ }^{+}$were run with selective coupling and coupled normalization.

- Case 1b: Same as Case 1a, but without coupled normalization.

- Case 1c: All components solved individually (SIA-1), without coupled normalization.

- Cases 2a-c: These runs employ identical solution strategies as Cases $1 \mathrm{a}-\mathrm{c}$, but the rate constants are an order of magnitude larger than those of Case 1.

The ion-exchange reactions in this example approach equilibrium behavior for Case 2 (Curve c in Fig. 4), but show significant deviations from equilibrium behavior for the slower kinetics of Case 1 (Curve b). Table 7 shows that the numerical performance of the coupled normalization and selective coupling method (Case 1a) was superior to either the selective coupling method without coupled normalization (Case 1b) or the SIA-1 method (Case 1c). In addition, if the kinetics are faster (Case 2), the performance degrades further for the cases without coupled normalization. Although it is difficult to compute a global value of $\mathrm{Da}$ for a complex system with varying velocities, the comparison between Cases 1 and 2 can be viewed in terms of the closeness to equilibrium reaction behavior. The more rapid the kinetics, the greater is the need to employ selective coupling and, in this example, especially coupled normalization. The $b$ and c cases only become competitive with the preferred coupling strategy (a) when the kinetics are slow enough 
Table 7

Computational efficiency, Neptunium transport problem

\begin{tabular}{|c|c|c|c|c|}
\hline Case & Description & $\begin{array}{l}\text { Total number } \\
\text { of time steps }\end{array}$ & $\begin{array}{l}\text { Total number } \\
\text { of iterations }\end{array}$ & $\begin{array}{l}\text { Total cpu } \\
\text { time (h) }\end{array}$ \\
\hline la & Selective coupling and coupled normalization, slow kinetics & 125 & 385 & 1.01 \\
\hline $1 b$ & Selective coupling only, slow kinetics & 486 & 2192 & 2.8 \\
\hline $1 \mathrm{c}$ & SIA-1, slow kinetics & 515 & 2433 & 2.39 \\
\hline $2 \mathrm{a}$ & Selective coupling and coupled normalization, rapid kinetics & 125 & 386 & 1.06 \\
\hline $2 b$ & Selective coupling only, rapid kinetics & 5379 & 20,103 & 25 \\
\hline $2 \mathrm{c}$ & SIA-1, rapid kinetics & 6463 & 24,005 & 23.3 \\
\hline
\end{tabular}

to result in significant deviations from equilibrium behavior. In Case 2, the b and c strategies can be made to produce results that are not significantly different from the coupled normalization case, but at great computational expense. Therefore, as in the previous examples, the numerical techniques developed in the present study are needed for the efficient solution of systems of chemical species coupled through kinetic reactions, especially for cases in which the reaction rates are rapid.

\section{Discussion and conclusions}

In this paper, we developed an iterative solution technique for reactive transport problems that represents a versatile alternative to traditional SIA techniques and the global implicit method. We note first that the term "coupling" refers to the strategy for solving the transport step of the iterative procedure, rather than the inherent coupled nature of the component concentrations. Regardless of the solution procedure, if convergence of the system of equations is achieved, subject to a prescribed tolerance criterion, the result is a valid solution to the coupled chemical transport system.

The SIA technique attempts to solve the linearized algebraic equations for transport of the aqueous components one at a time. This approach, though simple to implement, is not always an efficient solution technique because it ignores the coupling between aqueous components linked through kinetic reactions. If the reactions that link the aqueous components are rapid, the SIA technique ignores important derivatives $\left(\Delta t \partial R_{i} / \partial C_{j}, i \neq j\right)$ in the Jacobian matrix of the full system of equations.

The global implicit approach includes all derivatives, making no approximations in the formulation of the Jacobian matrix. Its performance is therefore controlled by the ability of the Newton-Raphson formulation to solve the system of nonlinear transport equations. This approach is likely to attain convergence efficiently for most cases. However, the global implicit method is very memory intensive for large problems with numerous aqueous components. The selective coupling method developed in the present study allows only the strongly coupled components to be solved together, and the transport iteration consists of solving groups of components simultaneously. This approach can result in computational savings relative to the global implicit method by achieving a similar total SIA iteration count while reducing the cpu time per iteration. More importantly, the memory requirements of the selective coupling technique are controlled by the maximum number of coupled components, rather than by the total number of components. For complex aqueous chemical systems and grids with a large number of nodes, the memory efficiency is the characteristic that makes the selective coupling method particularly attractive relative to the global implicit method.

There are many considerations to be made when developing a numerical technique for solving reactive transport problems. In our case, a flexible solution technique was desirable because the code was being developed for an existing, general-purpose finite element heat and mass transport code (FEHM). In the selective coupling method as implemented, the user selects the groups of solutes and the order in which the groups are solved. All possible coupling strategies, from the SIA-1 method to the global implicit technique, are available at run time. With respect to the solution of the coupled equations, the block matrix solver technology already used in the FEHM code is ideally suited for this application. Equation solvers of this type are fairly common, and hence availability should not be a roadblock to implementing these techniques in other codes.

Coupled normalization is another concept introduced in this paper for formulating the solution of the linearized equations resulting from the Newton-Raphson technique. Coupled normalization scales the equations in preparation for an iteration of the linear equations. Furthermore, we demonstrated how coupled normalization allows for the efficient coupling of aqueous and immobile components such that the computational and memory resources are dependent only on the number of coupled aqueous components; the immobile components are included with very low additional computational burden.

The choice of a chemical formulation with a combination of equilibrium and kinetic reactions results in a 
challenging system for obtaining an efficient numerical solution, but at the same time is a more versatile model formulation than a purely equilibrium-based system. Although it could be argued that the most computationally challenging problems for the SIA technique systems with rapid kinetic reactions - could be recast more efficiently as equilibrium reactions, there are several reasons that the more flexible system and the selective coupling solution procedure are desirable. First, geochemical rock-water reactions span an extremely large range of reaction rates, as do fluid velocities in porous media. Therefore, in practical applications a kinetic treatment is useful in many cases. Next, in many chemical systems there is scant knowledge on the kinetic parameters of the reactions. While no substitute for data, a sensitivity analysis in which kinetic parameters are varied over a wide range can provide insight into whether the lack of data is critical to understanding the system, or merely an uncertainty that is relatively unimportant to resolve. For this situation, the reactions in question are most conveniently cast as kinetic reactions; when large rate constants are selected the system behaves as an equilibrium system. This is preferable to having to recast the problem to handle the rapid-kinetics extreme. A final advantage of the flexibility of selective coupling method is that in more complex flow and transport systems, a reaction can be effectively at equilibrium in one part of the system and kinetically controlled in another part of the domain. This situation can occur in systems with large temperature or concentration gradients, or contrasting flow velocities or solid surface areas. A kinetic formulation and the selective coupling method is a versatile solution procedure for such systems. All of these reasons relate to the "robustness" of the numerical solution, which we define as the ability to obtain convergence for a wide range of input parameters in a reasonable time, without the need for intervention by the user once the problem is set up. Robustness is a particularly important attribute for a general purpose chemical transport model: if a solution cannot be obtained practically for some sets of parameters, then issues of cpu and memory efficiency are moot.

Examination of the example problems presented here allows us to formulate general guidelines for the selection of a coupling strategy for practical problems. The following guidelines are supported by the example problems.

(1) The most important aspect of the chemical system is the nature of the linking of aqueous component concentrations. Example 1 illustrated this concept by dealing only with aqueous components coupled through kinetic reactions. This example was actually a surrogate for more complex chemical systems with aqueous components linked indirectly through interphase kinetic reactions. The Damköhler numbers of the reactions strongly influenced which coupling strategy was the most appropriate.

(2) Linking of aqueous components can also occur indirectly through competitive sorption reactions or dissolution/precipitation reactions, and must be considered in selecting the coupling strategy. Example 2 illustrated both the concept of the influence of kinetics and the indirect linking of aqueous components through competitive reactions. Specifically, we showed that selectively coupling $\mathrm{Co}^{2+}$ and $\mathrm{NTA}^{3-}$ greatly improved convergence. Although $\mathrm{Co}^{2+}$ and $\mathrm{NTA}^{3-}$ do not react with one another in a kinetic reaction, they were indirectly linked to one another due to the equilibrium speciation reactions. For this reason, coupling of the two components improved convergence.

(3) The aqueous component concentrations themselves affect the nature of the chemical system and the optimal solution strategy. In Example 1, lowering the concentration of an aqueous component from a value at which it was in excess relative to the other concentrations to a value that was of the same order as the others made the solution more difficult to obtain without using a fully coupled strategy. The detailed knowledge required to optimally configure the solution strategy can perhaps be viewed as a limitation. However, it is just such an analysis that provides fundamental insight into the controlling factors of the behavior of the chemical system. Thus, we view the "burden" of gaining this insight as an advisable preliminary step in the analysis of a complex chemical transport system. In practice, this preliminary work can be carried out in simplified, steady-state, uniform flow and transport fields before progressing to more realistic scenarios.

(4) Coupled normalization results in a guaranteed memory savings when coupling immobile and mobile components, and hence should be employed whenever possible. In Example 3, the competitive ion exchange reaction presented a computationally demanding problem, especially for the more rapid kinetics. As rate constants increase, the most efficient strategy is to couple the three aqueous components and three immobile components. The coupled normalization approach couples the immobile components to the aqueous components in a way that effectively reduces the coupled solution from six to three degrees of freedom. The three aqueous components, with Jacobian derivative information from the three immobile components folded into the residual equations, were then solved as a system. The other components in this problem, $\mathrm{H}^{+}$and $\mathrm{HCO}_{3}^{-}$, were solved individually before the coupled solution step, thereby optimizing the computational and memory efficiency of the overall solution.

(5) The user should employ the minimum amount of coupling needed to solve a given problem efficiently. The global implicit method is the most computationally demanding option on a per-iteration basis, and thus will 
underperform a strategy using an intermediate coupling, as long as the latter solves the equations in a similar number of iterations. Furthermore, for large systems, the memory demands associated with high degrees of coupling are potentially prohibitive. The memory requirement of the transport solution is governed by the maximum number of components coupled in the iterative scheme.

The flexibility of the techniques developed in the present study provides a great advantage in that the solution strategy can be tailored to the problem at hand. As reactive transport simulations begin to become more commonly performed for complex, multi-dimensional flow and transport systems, we believe that the numerical techniques developed in the present study should find widespread applicability in general purpose reactive transport codes.

\section{Acknowledgements}

This work was supported by the Yucca Mountain Site Characterization Project Office as part of the Civilian Radioactive Waste Management Program. The Project is managed by the U.S. Department of Energy, Yucca Mountain Site Characterization Project. We thank Caroline Tebes-Stevens for helping us with setting up Example 2. We would also like to thank George Zyvoloski for helpful discussions during the development of the numerical techniques. Finally, we would like to thank Peter Lichtner and several anonymous reviewers for their helpful comments.

\section{References}

[1] Behie A, Collins D, Forsyth PA, Sammon PH. Fully coupled multiblock wells in oil simulation. SPE J 1985;535-42.

[2] Brusseau ML, Jessup RE, Rao PSC. Modeling the transport of solutes influenced by multiprocess nonequilibrium. Water Resour Res 1989;25:1971-88.

[3] Cederberg GA, Street RL, Leckie JO. A groundwater mass transport and equilibrium chemistry model for multicomponent systems. Water Resour Res 1985;21(8):1095-104.

[4] Engesgaard P, Kipp KL. A geochemical model for redoxcontrolled movement of mineral fronts in ground-water flow systems: a case of nitrate removal by oxidation of pyrite. Water Resour Res 1992;28:3308-27.

[5] Efurd DW, Runde W, Banar JC, Roensch FR, Clark DL, Palmer $\mathrm{PD}$, Tait $\mathrm{CD}$. Measured solubilities and speciation of neptunium and plutonium in J-13 groundwater, Los Alamos National Laboratory Yucca Mountain Site Characterization Project Milestone 3411, 1996.

[6] Goltz MN, Roberts PV. Interpreting organic solute transport data from a field experiment using physical nonequilibrium models. J Contam Hydrol 1986;1:77-94.

[7] Hutzler NJ, Crittenden JC, Gierke JS, Johnson AS. Transport of organic compounds with saturated groundwater flow: experimental results. Water Resour Res 1986;22:285-95.
[8] Kinzelbach W, Schafer W, Herzer J. Numerical modeling of natural and enhanced denitrification processes in aquifers. Water Resour Res 1991;27:1123-35.

[9] Lasaga AC. Chemical kinetics of water-rock interactions. J Geophys Res 1984;89:4009-25.

[10] Lichtner PC. Continuum formulation of multicomponent-multiphase reactive transport. Rev in Mineralogy 1996;34(1):1-81.

[11] Liu CW, Narasimhan TN. Redox-controlled- multiple-species reactive chemical transport, model development. Water Resour Res 1989;25(1):869-82.

[12] Nicoud RM, Schweich D. Solute transport in porous media with solid-liquid mass transfer limitations: application to ion exchange. Water Resour Res 1989;25:1071-82.

[13] Rittmann BE, Van Briesen JM. Microbial processes in reactive modeling. Rev in Mineralogy 1996;34(7):311-32.

[14] Rubin J. Transport of reacting solutes in porous media: relation between mathematical nature of problem formulation and chemical nature of reaction. Water Resour Res 1983;19:1231-52.

[15] Saad Y, Schultz MH. GMRES: A generalized minimum residual algorithm for solving non-symmetric linear systems. SIAM J Sci and Stat Comput 1986;7(3):856-69.

[16] Steefel CI, Lasaga AC. A coupled model for transport of multiple chemical species and kinetic precipitation/dissolution reactions with application to reactive flow in single phase hydrothermal systems. Amer J Sci 1994;294:529-92.

[17] Steefel CI, MacQuarrie TB. Approaches to modeling of reactive transport in porous media. Rev in Mineralogy 1996;34(2):83-125.

[18] Suarez DL, Simunek J. Solute transport modeling under variably saturated water flow conditions. Rev in Mineralogy 1996;34(5):229-64.

[19] Tebes-Stevens C, Valocchi AJ, Van Briesen JM, Rittmann BE. Multicomponent transport with coupled geochemical and microbiological reactions: model description and example simulations. J Hydrol 1998;209:8-26.

[20] Tebes-Stevens C, Valocchi AJ. Numerical solution techniques for reaction parameter sensitivity coefficients in multicomponent subsurface transport models, Hydraulic Engineering Series No. 59, Department of Civil and Environmental, University of Illinois, UIUC-ENG-98-2009, 1998.

[21] Tompson AFB, Jackson KJ. Reactive transport in heterogeneous systems: an overview. Rev in Mineralogy 1996;34(6):269-308.

[22] Valocchi AJ, Malmstead M. Accuracy of operator splitting for advection-dispersion-reaction problems. Water Resour Res 28:1471-76.

[23] Zee van der, Lens SF, Lauer M. Prediction of phosphate transport in small columns with an approximate sorption kinetics model. Water Resour Res 1989;25:1353-65.

[24] Viswanathan HS. Modification of the finite element heat and mass transfer code (FEHMN) to model multicomponent reactive transport, MS thesis, University of Illinois, Champaign-Urbana, Illinois, September, 1995, Technical Report LA-13167-T, Los Alamos National Laboratory, 1996.

[25] Viswanathan HS, Robinson BA, Valocchi AJ, Triay I. A reactive transport model of neptunium migration from the potential repository at Yucca Mountain. J Hydrol 1998;209:251-80.

[26] Wood WW, Kraemer TF, Hearn PP. Intragranular diffusion: an important mechanism influencing solute transport in clastic aquifers? Science 1990;247:1569-72.

[27] Wolery TJ. EQ3NR, A Computer Program for Geochemical Aqueous Speciation-Solubility Calculations: Theoretical Manual, User's Guide, and Related Documentation (Version 7.0), Technical Report UCRL-MA-110662-PT-IV, Lawrence Livermore National Laboratory, 1992.

[28] Yeh GT, Tripathi VS. A critical evaluation of recent developments in hydrogeochemical transport models of reactive multichemical components. Water Resour Res 1989;25(1):93-108. 
[29] Zyvoloski GA. Finite element methods for geothermal reservoir simulation. Int J Numer Meth Geomech 1983;7:75-86.

[30] Zyvoloski GA. Incomplete factorization methods for finite elements. Int J Numer Meth Eng 1986;23:1101-9.
[31] Zyvoloski GA, Robinson BA, Dash ZV, Trease LL. Summary of the Models and Methods for FEHM Application - A FiniteElement Heat- and Mass-Transfer Code, Technical Report LA13307-MS, Los Alamos National Laboratory 1997. 